\title{
Assessment of the severity of mitral regurgitation from the dynamics of retrograde flow
}

\author{
JULIE A BRADLEY, DEREK G GIBSON \\ From the Department of Cardiology, Brompton Hospital, London
}

SUMMARY Sixty four consecutive patients with isolated mitral regurgitation referred for Doppler? echocardiography were divided into three groups: group 1, 20 patients with severe mitrabo regurgitation that required operation; group 2,22 patients with severe left ventricular dysfunction and secondary mitral regurgitation; and group 3, 22 patients with mild to moderate mitra! regurgitation that did not require valve operation. $M$ mode and continuous wave Doppler traces ${ }^{+}$ with a simultaneous electrocardiogram and phonocardiogram were analysed to identify time $\mathrm{e}^{-}$ intervals that could be used to distinguish patients who needed valve operation from those who did not. An interval of $<55 \mathrm{~ms}$ between the aortic component of the second heart sound $\left(\mathrm{A}_{2}\right)$ and the्ष cessation of mitral retrograde flow was a powerful predictor that the patient required operationes (sensitivity $100 \%$ and specificity $86 \%$ ). The mean (SD) value of this variable in group $1(40(15))_{0}$ $\mathrm{ms}$ ) was significantly lower than in group $2(90(35) \mathrm{ms})$ and group $3(75(20) \mathrm{ms})$. Mean isovolumico. relaxation time was less than normal in group 1 but did not differ significantly between groups. Deceleration of regurgitant velocity at end ejection was greater in group 1 . The pressure drop from? the left ventricle to the left atrium at $A_{2}$ of $<50 \%$ of the peak gradient also identified patients who needed valve operation (sensitivity $75 \%$ and specificity $68 \%$ ).

These findings may help to identify patients who require operation. They suggest that there are significant differences in the dynamics of flow velocities in patients with mitral regurgitation $\vec{s}$ possibly related to the relative resistances to retrograde and anterograde flow.

The severity of mitral regurgitation is an important factor in determining the need for valve operation. There are technical limitations with all current methods of quantification including contrast ventriculography, which has generally been regarded as the reference standard. ${ }^{1-4}$ Doppler echocardiography, though widely used to detect mitral regurgitation, ${ }^{5}$ is less well established as a method of determining its severity. Pulsed Doppler has been used to map the extent of regurgitation into the left atrium. ${ }^{67}$ This procedure is time consuming and may be misleading, particularly when an eccentric jet caused by mitral valve prolapse extends far back into the left atrium without severe regurgitation. Similarly, determination of regurgitant fraction with pulsed Doppler also has limitations because it is necessary to estimate the mitral valve area and flow profile or because there is additional valve regurgitation at the aortic or tricuspid valve. ${ }^{89}$

Requests for reprints to Dr Derek G Gibson, Brompton Hospital, Fulham Road, London SW3 6HP.

Accepted for publication 24 February 1988
The present study arose out of an examination of the relation between events documented by $M$ mode and continuous wave Doppler tracings of patients? with mitral regurgitation. Particular attention was paid to the duration of mitral regurgitant flow aftern end ejection and to the contour of the terminas portion of the Doppler profile. The aim was to identify time intervals that might distinguish? patients who required valve operation from those who did not.

\section{Patients and methods}

PATIENTS

All patients with mitral regurgitation referred fof Doppler echocardiography between January 1986 and January 1987 were studied retrospectively. We excluded patients with other important valve disease We did not need to exclude any patients because we्ष were unable to obtain Doppler echocardiographiष्षि measurements. The standard of comparison that we used was that of "outcome", namely whether or nok 
mitral valve operation was advised. The clinical criteria for valve operation included: $(a)$ limitation of exercise tolerance; $(b)$ physical signs compatible with severe mitral regurgitation, including a third heart sound; (c) radiological evidence of cardiac enlargement and pulmonary congestion; $(d)$ echocardiographic evidence of increased left ventricular cavity size and stroke volume along with anatomical abnormalities of the mitral valve.

The 64 patients studied belonged to three groups. Group 1 comprised those patients with severe mitral regurgitation and who required operation. Group 2 consisted of patients with mitral regurgitation secondary to severe left ventricular disease, and group 3 consisted of patients with mild or moderate primary mitral regurgitation in whom operation was not advised. There were 20 patients (11 men, nine women, mean (SD) age 60 (17)) in group 1. The cause of mitral regurgitation was flail chordae tendineae in nine $(45 \%)$, mitral valve prolapse in six $(30 \%)$, rheumatic disease in two $(10 \%)$, papillary muscle infarction in two $(10 \%)$, and endocarditis in one $(5 \%)$. Mitral valve repair was performed in 10 and mitral valve replacement in eight. Two patients are awaiting operation. In all cases the findings at operation were those of severe mitral regurgitation. Group 2 comprised 22 patients ( 16 men, six women, mean (SD) age $64(14 \cdot 8)$ ). Eleven had ischaemic left ventricular disease with papillary muscle dysfunction and 11 had dilated cardiomyopathy. The remaining 22 patients (18 men, four women, mean age 60 (17)) comprised group 3. Ten (45\%) had mitral valve prolapse and three of these developed endocarditis; four $(18 \%)$ had papillary muscle dysfunction; three (14\%) had rheumatic mitral regurgitation; one ( $4.5 \%$ ) had flail chordae tendineae; one $(4.5 \%)$ had annular calcification, and there was no clear cause in three $(14 \%)$.

\section{ECHOCARDIOGRAPHY}

$M$ mode and cross sectional echocardiograms were obtained with an Advanced Technical Laboratory $860 \mathrm{C}$ Imager equipped with a $3.5 \mathrm{MHz}$ mechanical transducer. $M$ mode echocardiograms were recorded on a Honeywell (Echoline 22) strip chart recorder at a paper speed of $100 \mathrm{~mm} / \mathrm{second}$. The system was linked to a Doptek Spectrascan, and continuous wave Doppler signals were similarly recorded at $100 \mathrm{~mm} /$ second paper speed, with a $2.0 \mathrm{MHz}$ transducer, at a range of frequency shift of $16 \mathrm{kHz}$. Recordings were made from the apex with the patient in the left lateral decubitus position. The delay in Doppler signal because of spectral analysis was $<5 \mathrm{~ms}$. A phonocardiogram at the second right interspace and an electrocardiogram were recorded simultaneously with both $M$ mode and Doppler recordings (figs 1 and 2). We used calipers to measure the following, as the mean value of three beats in patients in sinus rhythm and 5-10 beats in those in atrial fibrillation:

(a) $\mathbf{R} \mathbf{R}$ interval and heart rate.

(b) End diastolic and end systolic dimensions measured synchronously with onset of $Q$ wave and $A_{2}$, respectively. Fractional shortening was derived as systolic reduction in dimension divided by end diastolic dimension.

(c) Left ventricular filling time-the interval between initial separation and final closure of the mitral leaflets at the beginning and end of diastole.

(d) Q-A $\mathrm{A}_{2}$ interval (electromechanical systole), taken from the onset of the $Q$ wave to the first high frequency deflection on the phonocardiogram. The identity of $\mathrm{A}_{2}$ was checked by confirming that it was synchronous with aortic closure on the aortic echogram.

(e) Isovolumic relaxation time, the interval $A_{2}$ to mitral valve opening. Records were obtained at the level of the tips of the mitral leaflets to display initial cusp separation.

$(f)$ Interval $A_{2}$ to the end of mitral systolic flow.

(g) Relative gradient at $A_{2}$, measured as the left ventricular to left atrial pressure difference at $A_{2}$, expressed as a percentage of the peak left ventricular to left atrial pressure difference.

(h) Duration of retrograde mitral systolic flow measured from the continuous wave Doppler trace.

Interobserver variability was small ( $<10 \mathrm{~ms})$. Beat to beat changes in end-systolic and early diastolic time intervals in patients with atrial fibrillation were also small, and within the $10 \mathrm{~ms}$ interval to which all measurements were rounded off.

\section{ANGIOGRAPHIC EXAMINATION}

Angiography was only performed in patients in whom there was doubt about the severity of mitral regurgitation after clinical assessment and noninvasive examination, or when concomitant coronary artery disease was suspected. In these patients right heart catheterisation was performed in addition to left ventricular cineangiography in the $30^{\circ}$ right anterior oblique projection. The angiographic severity of mitral regurgitation was classified as follows: $1+$, contrast cleared the left atrium with each cardiac cycle; $2+$, contrast partially cleared the left atrium with each cardiac cycle; $3+$, contrast progressively opacified the left atrium so that it equalled the opacification of the left ventricle and $4+$, contrast rapidly opacified the left atrium within 1 to 2 cycles and opacification became greater than in the left ventricle and extended to the pulmonary veins. 


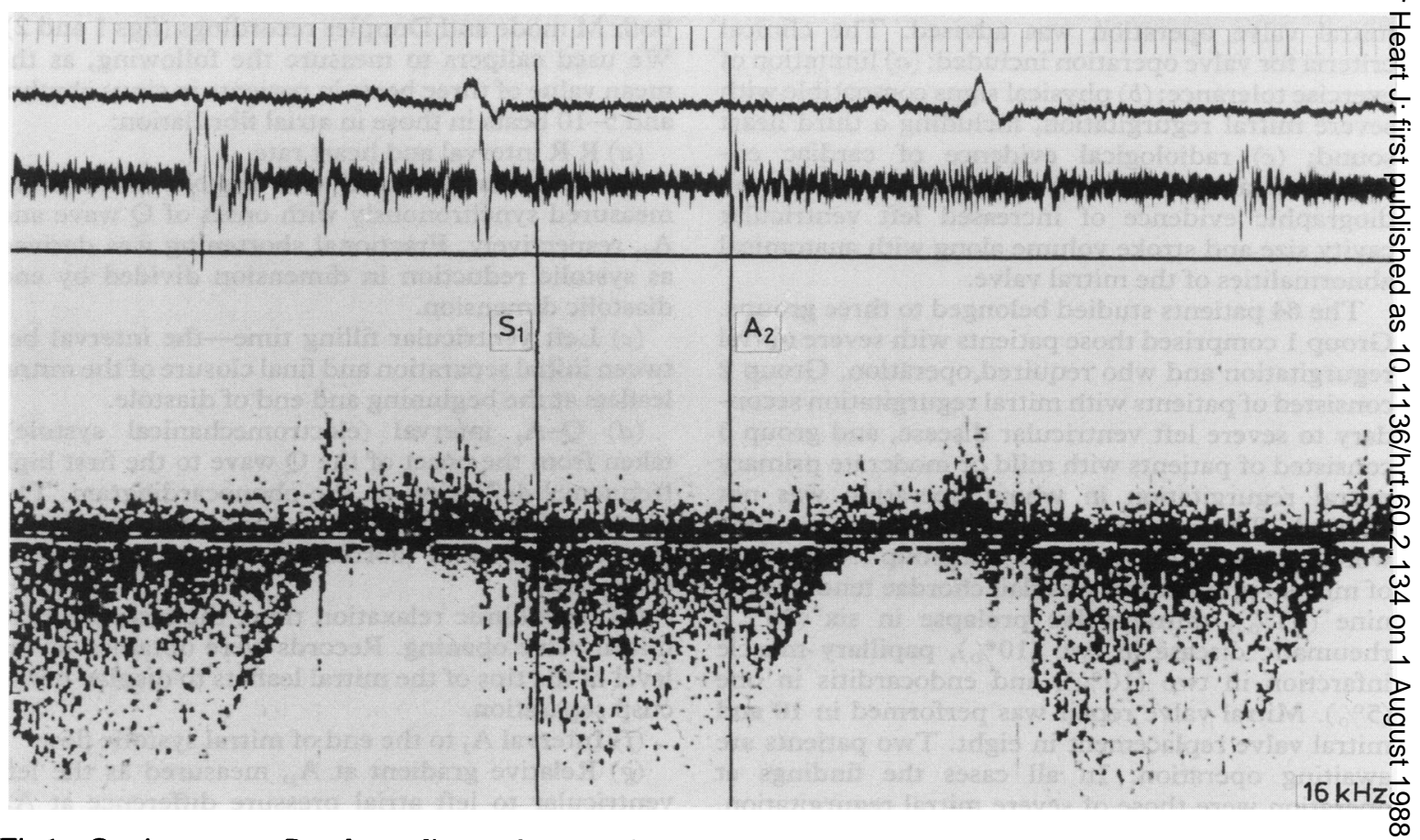

Fig 1 Continuous wave Doppler cardiogram from a patient with functional mitral regurgitation (recorded with simultaneous phonocardiogram). Mitral flow continued for $140 \mathrm{~ms}$ after $A_{2}$, the aortic component of the second heart sound. S1, first heart sound. Full scale deflection $=16 \mathrm{kHz}$ frequency shift, equivalent to a velocity of $6.4 \mathrm{~m} / \mathrm{s}$.

\section{STATISTICAL ANALYSIS}

The mean (1SD) was calculated for each measurement in each group and compared by the paired $t$ test.

\section{Results}

Table 1 summarises the clinical features. There was no significant difference between the three groups in age or heart rate, or between groups 1 and 2 in clinical or radiological manifestations of clinical severity or pulmonary congestion. Table 2 shows the echocardiographic estimates of left ventricular function. These reflected the expected differences between the groups. There was no significant difference between groups 1 and 3 for end systolic and end diastolic cavity size, though fractional shortening was significantly increased in group $1(p<0.05)$. There was no significant difference between any of the groups in isovolumic relaxation time; this was significantly shorter than normal $(60(10) \mathrm{ms})$ in all three. The values for groups 1 to 3 were 20 (25) ms, 30 (30) ms, and $30(40) \mathrm{ms}$ (all p $<0.001$ compared with the normal value). The mean interval from $A_{2}$ to end of mitral retrograde flow was significantly shorter in group 1 than in group $2(\mathrm{p}<0.001)$ or group 3 (p $<\frac{2}{\Phi}$ $0.001)$. The mean of group 2 was significantly less than that of group $3(p<0.05)$. In all patients with mitral regurgitation severe enough to require opera- $-\frac{3}{5}$ tion, this interval was $<55 \mathrm{~ms}$; the mean value in group 1 was 40 (15) ms compared with 90 (35) ms in group 2, and $75(20) \mathrm{ms}$ in group 3 . The sensitivity, specificity, and predictive accuracy of an interval $\stackrel{\varrho}{7}$ $\leqslant 55 \mathrm{~ms}$ in predicting the need for valve operation was $100 \%, 86 \%$, and $91 \%$, respectively. There were. three false positives in group 3 , in whom the intervals from $A_{2}$ to cessation of mitral flow were 45,45 , and $53 \mathrm{~ms}$. The first patient has angiographic grade $4+0$ mitral regurgitation but is only mildly limited, a. $\mathrm{a}$ is being observed for symptomatic deterioration before operation is recommended. The other two had moderately impaired left ventricular function with $\mathrm{N}$ clinically mild to moderate mitral regurgitation and $N$ have not been catheterised. There were three false N్ positives in group 2 but all had dilated car- $\sigma$ diomyopathy with severely reduced fractional shortening $(9 \%, 14 \%$, and $14 \%)$. They did not appear to have severe mitral regurgitation clinically and could $\stackrel{\oplus}{\oplus}$ be readily distinguished from group 1 .

The relative gradient at $A_{2}$ was also significantly 


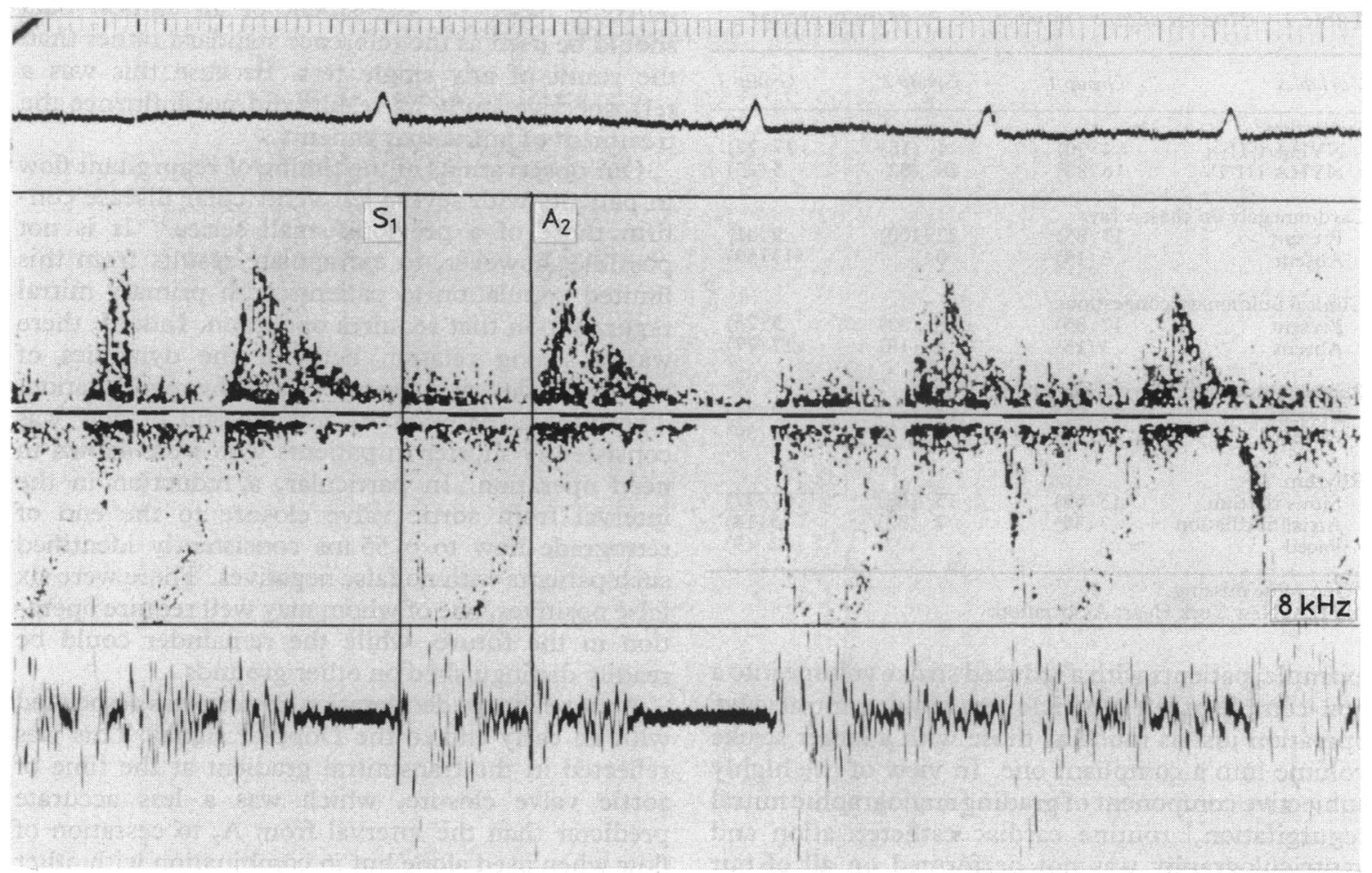

Fig 2 Continuous wave Doppler cardiogram from a patient with severe mitral regurgitation caused by ruptured posterior chorda tendineae. The end of mitral regurgitant flow was synchronous with $A_{2}$. Full scale deflection $=8 \mathrm{kHz}$, equivalent to a velocity of $3.2 \mathrm{~m} / \mathrm{s}$. Note also cessation of regurgitant murmur in mid-systole.

lower in group $1(35(15) \%)$ than in group $2(55$ $(20) \%)(p<0.005)$ and group $3(60(20) \%)$ (p < $0.001)$. A ratio of $<50 \%$ identified those who required valve operation, with a sensitivity of $75 \%$ and a specificity of $68 \%$. The correlation between isovolumic relaxation time and the interval from $A_{2}$ to end of mitral retrograde flow was weak, with correlation coefficients of $0.24,0.45$, and 0.58 , respectively. That between $A_{2}$ to cessation of flow (x) and relative gradient at $A_{2}$ (y) was 0.57 , with a regression equation of $y=0.37 x+24$, and the standard error of the estimate $16 \%$. When a combination of $A_{2}$ to cessation of flow of $<55 \%$ and a relative gradient at $\mathrm{A}_{2}$ of $<50 \%$ was used, the specificity rose to $90 \%$ and the sensitivity remained at $100 \%$. Table 3 summarises the mean values for the systolic time intervals and left ventricular filling time. The $Q-A_{2}$ interval was significantly shorter in group 1 but this was of no predictive accuracy in individual patients.

Left ventricular angiography was performed in eight ( $40 \%$ ) out of 20 group 1 patients, seven had $4+$ mitral regurgitation. The remaining patient was considered to have $3+$ mitral regurgitation and after several months of symptomatic deterioration had mitral valve repair without recatheterisation. Three group 2 patients underwent ventriculography and were shown to have $1+, 2+$, and no mitral regurgitation. In group 3, five patients were studied angiographically; there was $2+$ mitral regurgitation in two, $3+$ in two, and $4+$ in the patient awaiting operation. Thus in the limited number of patients studied, the correlation between angiographic and Doppler assessment of mitral regurgitation was close.

\section{Discussion}

It is difficult to compare different methods of measuring mitral regurgitation because there is no reference standard. ${ }^{1011}$ Volume measurements such as cardiac output, left ventricular volume, ejection fraction, or regurgitation fraction and pressure measurements including pulmonary arterial and wedge pressures, all provide ancillary information but seem to be intrinsically limited as indicators of the appropriate timing of mitral valve operation. In addition to technical limitations, this may be partly because of difficulties in identifying additional complications caused by left ventricular disease. For 
Table 1 Clinical details (number (\%)) of study groups

\begin{tabular}{lcrr}
\hline Variables & Group I & Group 2 & Group 3 \\
\hline $\begin{array}{l}\text { Symptoms: } \\
\text { NYHA I/II }\end{array}$ & $4(20)$ & $4(18)$ & $17(77)$ \\
NYHA III/IV & $16(80)$ & $18(82)$ & $5(23)$ \\
Cardiomegaly on chest & $x$ ray: & & \\
$\begin{array}{l}\text { Present } \\
\text { Absent }\end{array}$ & $17(85)$ & $22(100)$ & $9(41)$ \\
Clinical pulmonary congestion: & $3(15)$ & 0 & $13(59)$ \\
$\begin{array}{l}\text { Present } \\
\text { Absent }\end{array}$ & $17(85)$ & $18(82)$ & $5(23)$ \\
Radiographic pulmonary congestion: & $3(15)$ & $4(18)$ & $17(77)$ \\
$\begin{array}{l}\text { Present } \\
\text { Absent }\end{array}$ & $17(85)$ & $17(77)$ & $9(41)$ \\
Rhythm: & $3(15)$ & $4(18) \star$ & $13(59)$ \\
$\begin{array}{l}\text { Sinus rhythm } \\
\text { Atrial fibrillation }\end{array}$ & $13(65)$ & $15(68)$ & $17(77)$ \\
Paced & $7(35)$ & $7(32)$ & $3(14)$ \\
\hline
\end{tabular}

^One value missing.

NYHA, New York Heart Association.

example, patients with a reduced stroke volume into a non-compliant left ventricle may require mitral valve operation just as much as those with a larger stroke volume into a compliant one. In view of the highly subjective component of grading angiographic mitral regurgitation, ${ }^{3}$ routine cardiac catheterisation and ventriculography was not performed on all of our patients. In many the diagnosis and haemodynamic severity of the mitral regurgitation was readily apparent by clinical and non-invasive assessment. Invasive investigations were only performed if there was some doubt about the severity of the lesion or because of the possibility of coexistent coronary artery disease, an approach that has been previously validated in a large series of patients. ${ }^{12}$ In all operated patients the surgical findings confirmed severe mitral regurgitation. Since the aim of investigation is to determine clinical management, we concluded that
Bradley, Gibsor $\stackrel{\frac{w}{7}}{\pi}$

outcome based on assessment of all clinical dată should be used as the reference standard rather tharf. the result of any single test. Because this was $\vec{F}^{*}$ retrospective study its results did not influence the treatment of individual patients.

Our observations of the timing of regurgitant flow: in patients with severe left ventricular disease con $\overline{\widehat{D}}$ firm those of a previous small series. ${ }^{13}$ It is no? possible, however, to extrapolate results from thiss limited population to patients with primary mitrab regurgitation that requires operation. Indeed, there was a strong relation between the dynamics of retrograde flow and the severity of the regurgitation 2 The interval between $A_{2}$ and the end of flow was consistently shorter in patients who were shown to need operation. In particular, a reduction in the interval from aortic valve closure to the end of retrograde flow to $<55 \mathrm{~ms}$ consistently identifie of such patients with no false negatives. There were six? false positives, one of whom may well require opera $\rightarrow$ tion in the future, while the remainder could bef readily distinguished on other grounds.

A more abrupt deceleration pattern was associated with an early end to the Doppler signal. This waso reflected in the transmitral gradient at the time of ${ }^{\circ}$ aortic valve closure, which was a less accurate predictar than the interval from $A_{2}$ to cessation of flow when used alone but in combination with othez̃ measurements seemed to provide additional infor: mation. The velocity profile in the non-surgicab group seemed to reach a peak in mid-ejection, but with severe regurgitation, there was an earlier peak that was more reminiscent of a normal aortic velocity? profile (fig 2). The two profiles wereeasily distinguis: shed because blood flow velocities caused by mitraf regurgitation, whatever their profile, are much higher than those of aortic flow if there is no aortic stenosis. To measure these time intervals $A_{2}$ must vbe unequivocally identified, especially in patients with

Table 2 Left ventricular cavity size (mean (SD)) in study groups

\begin{tabular}{|c|c|c|c|c|c|c|}
\hline Measurement & Group 1 & Group 2 & Group 3 & $1 \vee 2$ & 103 & $2 v 3$ \\
\hline $\begin{array}{l}\text { End systolic dimension (mm) } \\
\text { End diastolic dimension (mm) } \\
\text { Fractional shortening (\%) }\end{array}$ & $\begin{array}{l}35(10) \\
60(10) \\
40(10)\end{array}$ & $\begin{array}{l}55(10) \\
65(10) \\
15(5)\end{array}$ & $\begin{array}{l}40(10) \\
60(10) \\
35(10)\end{array}$ & $\begin{array}{l}p<0.001 \\
\mathrm{NS} \\
\mathrm{p}<0.001\end{array}$ & $\begin{array}{l}\mathbf{N S} \\
\mathbf{N S} \\
\mathbf{p}<0.05\end{array}$ & $\begin{array}{l}\mathrm{p}<0.001 \mathrm{~N} \\
\mathrm{NS} \\
\mathrm{p}<0.001 \mathrm{~N}\end{array}$ \\
\hline
\end{tabular}

Table 3 Left ventricular function (mean (SD)) in the study groups

\begin{tabular}{|c|c|c|c|c|c|c|}
\hline Interval & Group 1 & Group 2 & Group 3 & 102 & $1 v 3$ & $2 v 3$ \\
\hline $\begin{array}{l}\text { RR interval (ms) } \\
\mathbf{A}_{2} \text { to cessation of flow (ms) } \\
\text { Relative gradient at } \mathbf{A}_{2}(\%) \\
\text { Isovolumic relaxation time (ms) } \\
Q-\mathbf{A}_{2} \text { interval (ms) } \\
\text { Left ventricular filling time (ms) } \\
\text { Duration of systolic mitral flow (ms) }\end{array}$ & $\begin{array}{rr}670 & (170) \\
40 & (15) \\
35 & (15) \\
20 & (25) \\
330 & (30) \\
330 & (95) \\
300 & (95)\end{array}$ & $\begin{array}{r}680(130) \\
90(35) \\
55(20) \\
30(30) \\
360(50) \\
400(85) \\
380(60)\end{array}$ & $\begin{array}{rr}730 & (160) \\
75 & (20) \\
60 & (20) \\
30 & (40) \\
365 & (45) \\
400 & (95) \\
340 & (84)\end{array}$ & $\begin{array}{l}\text { NS } \\
\mathbf{p}<0.001 \\
\mathbf{p}<0.005 \\
\mathbf{N S} \\
\mathbf{p}<0.001 \\
\mathbf{p}<0.05 \\
\mathbf{p}<0.001\end{array}$ & $\begin{array}{l}\text { NS } \\
p<0.001 \\
p<0.001 \\
\text { NS } \\
p<0.001 \\
p<0.05 \\
\text { NS }\end{array}$ & 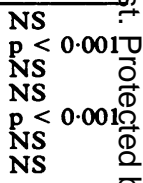 \\
\hline
\end{tabular}


left bundle branch block or paced rhythm in whom an error might be caused by a delay in $A_{2}$. Conversely, $A_{2}$ itself may occur early and be soft when mitral regurgitation is severe. The timing of aortic valve closure must therefore be routinely confirmed from a concomitant aortic echogram.

The advantages of the present technique were its ease and relative simplicity. We found no patient in whom the interval $A_{2}$ to cessation of retrograde flow could not be measured and only one in whom it was impossible to obtain the relative gradient at $A_{2}$. The method is equally applicable to patients with atrial fibrillation. In addition, it was unnecessary to measure the areas of the aortic or mitral orifices, as is required for Doppler determination of regurgitant fraction. Incomplete opening of the aortic valve followed by premature closure, seen in several of our patients may well prevent reliable estimates of aortic flow from velocity determinations. Derivation of mitral orifice area requires computer linked planimetry and is influenced by limited lateral resolution of echocardiographic equipment used for cross sectional imaging and the pattern of leaflet movement. Not surprisingly, Doppler assesssment often shows "regurgitant fractions" of $20 \%$ in normal valves. Although the degree of mitral regurgitation can be estimated by mapping the regurgitant flow in the left atrium, this can be significantly overestimated or underestimated. The method is very time consuming when simple pulsed Doppler is used. Colour flow mapping speeds up the investigation; however, with this technique small alterations in transducer angulation and subtle gain setting modifications also lead to uncertainties. ${ }^{14}$ The present method is based on relative velocities and time intervals and so is not sensitive to transducer angulation.

In contrast to the consistent relation between the need for valve operation and the length of the interval from $A_{2}$ to cessation of mitral retrograde flow, we found no correlation between the clinical or radiological evidence of pulmonary congestion and the isovolumic relaxation period. This might seem to be paradoxical, because the height of the left atrial " $v$ " wave is related to the severity of regurgitation and raised left atrial pressure is associated with a short isovolumic relaxation time both in left ventricular disease ${ }^{15}$ and mitral regurgitation. The interval $\mathrm{A}_{2}$ to cessation of retrograde mitral flow was short only in group 1 patients and this must therefore reflect some other aspect of the disordered physiology of mitral regurgitation. When regurgitation is severe, functional end systolic volume seems to be achieved earlier. This suggestion was supported in the present study by the $Q-A_{2}$ interval being significantly shorter in group 1 than either groups 2 or 3, with several patients showing early partial aortic closure even before $A_{2}$. The similarity between the aortic and mitral velocity profiles, both in form and timing, in patients with severe mitral regurgitation suggests that a major determinant of retrograde flow is the overall resistance to regurgitation and its relation with that to forward flow. Conditions thus seem similar to those governing ejection in patients with a large ventricular septal defect, where flows are neither determined nor predicted by pressures in the two great arteries, but depend more closely on the relative resistances.

We conclude that analysis of flow dynamics may be helpful not only in determining severity in individual cases, when used in conjunction with clinical features, but also seem to provide some insight into the pathophysiology of the regurgitation itself. Electromechanical systole and flow rate must be in balance. In severe mitral regurgitation this relation is likely to be severely disturbed, with low resistance to ejection being accompanied by a very rapid fall in left ventricular volume. Thus it seems that aspects of mitral regurgitation other than regurgitant fraction and pressure equalisation should be investigated when such patients are being assessed for operation. The idea of relative left atrial and aortic resistance as a determinant of flow may thus provide insight into the physiological disturbances determining flow and so improve the management of patients with severe mitral regurgitation.

This study was supported in part by grants from the E R Dawes, A R Curren, L C Hughes Research Fellowship provided by Royal Adelaide Hospital, from the National Heart Foundation of Australia, and from the Special Cardiac Fund, Brompton Hospital.

\section{References}

1 Honey M, Gough JH, Katsaros S, Miller GAH, Thuraisingham $V$. Left ventricular cine-angiocardiography in the assessment of mitral regurgitation. Br Heart J 1969;31:596-602.

2 Baron MG. Angiographic evaluation of valvular insufficiency. Circulation 1971;43:599-605.

3 Lopez JF, Hanson S, Orchard RC, Tan L. Quantification of mitral valvular incompetence. Cathet Cardiovasc Diagn 1985;11:139-52.

4 Urquhart J, Patterson RE, Packer M, et al. Quantification of valve regurgitation by radionuclide angiography before and after valve replacement surgery. $\mathrm{Am} \mathrm{J}$ Cardiol 1981;47:287-91.

5 Adhar GC, Nanda NC. Doppler echocardiography, Part II. Adult valvular heart disease. Echocardiogr 1984;1:219-41.

6 Quinones MA, Young JB, Waggoner AD, Ostojic MC, Ribeiro LGT, Miller RR. Assessment of pulsed Doppler echocardiography in detection and quan- 
tification of aortic and mitral regurgitation. Br Heart $J$ 1980;44:612-20.

7 Veyrat C, Ameur A, Bas S, Lessana A, Abitbol G, Kalmanson D. Pulsed Doppler echocardiographic indices for assessing mitral regurgitation. Br Heart $J$ 1984;51:130-8.

8 Rokey R, Sterling L, Zoghbi W, et al. Determination of regurgitant fraction in isolated mitral or aortic regurgitation by pulsed Doppler. J Am Coll Cardiol 1986;7:1273-8.

9 Blumlein S, Bouchard A, Schiller N, Dae M, Byrd B, Ports T, Botvinick E. Quantification of mitral regurgitation by Doppler echocardiography. Circulation 1986;74:306-14.

10 Croft C, Lipscomb K, Mathis K, et al. Limitations of quantitative angiographic grading in aortic or mitral regurgitation. Am J Cardiol 1984;53:1593-8.

11 Hochreiter C, Niles N, Devereux RB, Kligfield P, Borer JS. Mitral regurgitation: relationship of noninvasive descriptors of right and left ventricular performance to clinical and hemodynamic findings and to prog- nosis in medically and surgically treated patientr. Circulation 1986;73:900-12.

12 St John Sutton MG, St John Sutton M, Oldershaw $P, \stackrel{C}{\stackrel{c}{e t}}$ al. Valve replacement without cardiac catheterization. N Engl J Med 1981;305:1233-8.

13 Keren G, LeJemtel TH, Zecler AA, Meisner JS, Bier Yellin EL. Time variation of mitral regurgitant flow in patients with dilated cardiomyopathy. Circulatiog 1986;74:684-92.

14 Miyatake $\mathrm{K}$, Izumi S, Okamoto $\mathrm{M}$, et al. Semiquaf titative grading of severity of mitral regurgitation by real-time two-dimensional Doppler flow imaging technique. J Am Coll Cardiol 1986;7:82-8.

15 Mattheos M, Shapiro E, Oldershaw PJ, Sacchetti Gibson DG. Non-invasive assessment of changes left ventricular relaxation by combined phonos, echo-, and mechanocardiography. $\mathrm{Br}$ Heart of 1982;47:253-60.

16 Gamble WH, Salerni R, Shaver JA. The noninvasite assessment of pulmonary capillary pressure in mitrîl regurgitation. Am Heart J 1984;107:950-8. 